\title{
Accuracy of FDG-PET/CT in Colorectal Peritoneal Carcinomatosis: Potential Tool for Evaluation of Chemotherapeutic Response
}

\author{
GABRIEL LIBERALE ${ }^{1}$, CÉLINE LECOCQ ${ }^{1}$, CAMILO GARCIA $^{2}$, KRISTOF MUYLLE $^{2}$, ANGÉLIQUE COVAS ${ }^{3}$, \\ AMÉLIE DELEPORTE $^{3}$, ALAIN HENDLISZ ${ }^{3}$, FIKRI BOUAZZA ${ }^{1}$, ISSAM EL NAKADI ${ }^{1}$ and PATRICK FLAMEN ${ }^{2}$ \\ Departments of ${ }^{1}$ Surgery, ${ }^{2}$ Nuclear Medicine and ${ }^{3}$ Medical Oncology, \\ Jules Bordet Institute, Free University of Brussels, Brussels, Belgium
}

\begin{abstract}
Background/Aim: Neoadjuvant chemotherapy may be administered to patients with peritoneal carcinomatosis $(P C)$ of colorectal cancer $(C R C)$ origin. This study evaluated the performance of ${ }^{18}$ fluorodeoxyglucose positron-emission tomography (FDG-PET)/computed tomography $(C T)$ in detection of $P C$ from $C R C$ and correlated the most metabolically active quadrant with the most affected peritoneal area determined during surgery. Patients and Methods: This retrospective study compared the performance of FDG-PET/CT for PC diagnosis in 26 patients with CRC with histopathologically-confirmed $P C$ with a control group of 26 patients. An FDG-PET/CT score established for each patient diagnosed with PC was compared with the peritoneal cancer index $(P C I)$ performed during surgery. Results: The sensitivity and specificity of FDG-PET/CT for PC detection were $85 \%$ (22/26) and $88 \%$ $(23 / 26)$, respectively. The most scored quadrant by FDG$P E T / C T$ corresponded to the most scored quadrant at surgery in $77.3 \%$. Conclusion: FDG-PET/CT may represent a useful tool for evaluating response to neoadjuvant chemotherapy in patients with PC of CRC origin.
\end{abstract}

Peritoneal carcinomatosis (PC) represents a dramatic stage of cancer evolution. The presence of $\mathrm{PC}$ is associated with a poor prognosis. The reported median survival for PC is 6 months for patients treated with palliative chemotherapy (1). PC is often associated with distant metastases. However, in $20-25 \%$ of

Correspondence to: Liberale Gabriel, Rue H. Bordet, 1, 1000 Brussels, Belgium. Tel: +32 26477634, Fax: +32 25413141, e-mail: gabriel.liberale@bordet.be

Key Words: Colorectal cancer, peritoneal carcinomatosis, FDG$\mathrm{PET} / \mathrm{CT}$, hyperthermic intraperitoneal chemotherapy, complete cytoreductive surgery, neoadjuvant chemotherapy, peritoneal cancer index, PCI. cases, particularly for those with gastrointestinal tumours, the disease is confined to the peritoneal cavity $(2,3)$. Complete cytoreductive surgery with hyperthermic intraperitoneal chemotherapy (HIPEC) allows some patients to be treated with curative intent (4-6). The reported global 5-year survival in selected patients with PC of colorectal cancer origin is $51 \%$ with a median survival of 63 months in a recent series (7).

Although there are limited data on neoadjuvant chemotherapy (NAC) in patients with limited and resectable disease, NAC is usually used in patients with more extensive disease or in those with borderline disease in an effort to make them better candidates for complete cytoreductive surgery. The evaluation of therapeutic response based strictly on descriptive imaging using computed tomography (CT) is difficult as it does not allow a precise description of the PC (8-11). Recently, several studies have reported the use of ${ }^{18}$ fluorodeoxyglucose positron-emission tomography (FDG-PET) combined with CT in the evaluation of early chemotherapeutic response of various tumour types $(12,13)$. Data concerning the efficacy of FDG$\mathrm{PET} / \mathrm{CT}$ in the evaluation of $\mathrm{PC}$ of colorectal cancer origin is sparse $(14,15)$. If the sensitivity of FDG-PET/CT is sufficient and a good correlation between the most metabolically active lesions and the most affected surgical area is observed, FDGPET/CT could be used as an imaging tool to evaluate and guide the therapeutic plan of patients with PC. For example, patients with advanced or borderline disease responding to chemotherapy could receive chemotherapy and then undergo surgery, while patients with limited or borderline disease nonresponsive to chemotherapy could undergo surgery immediately.

The primary aim of this study was to evaluate the sensitivity, specificity, and accuracy of FDG-PET/CT in the diagnosis of PC of colorectal cancer origin. The secondary aim was to evaluate the correlation between the most metabolically active lesion (or most extended) on FDG$\mathrm{PET} / \mathrm{CT}$ imaging with the extent of intraoperative peritoneal dissemination. 


\section{Patients and Methods}

This was a retrospective study including patients selected from a surgical database (HIPEC protocol) who underwent surgical exploration for PC of colorectal origin and who underwent preoperative FDG-PET/CT during the work-up. The study included a control group of patients selected from an institutional computerized database $\left(\right.$ Oribase $\left.^{\circledR}\right)$. A database search was performed for non-metastatic colorectal cancer and PET/CT. This study was approved by the Ethics Committee of our Cancer Comprehensive Center, with the reference number B07920095814.

Study population. Group A included 26 patients with PC of colorectal origin, confirmed by histopathology performed during surgery, who underwent preoperative FDG-PET/CT. The delay between FDG-PET/CT and cytoreductive surgery was less than six weeks. Group B (control group), included 26 patients with colorectal cancer who had undergone FDG-PET/CT for various clinical reasons, and who had no signs of PC during a follow-up of at least 1 year following the PET. These FDG-PET/CT scans were performed in a preoperative $(n=6)$ or postoperative $(n=20)$ setting of the primary tumour.

Cytoreductive surgery. Patients were positioned in a supine position with legs extended and in abduction. The surgery was conducted via a xypho-pubian incision. Before performing the surgical resection, a systematic explorative laparotomy was performed. Evaluation of peritoneal disease was performed using the peritoneal cancer index (PCI) defined by Jacquet and Sugarbaker (16). Patients were included in our protocol for cytoreductive surgery and HIPEC if they presented with complete macroscopic resectable disease with a PCI $\leq 23$. The technical aspects used for the performance of cytoreductive surgery and HIPEC were described previously (17). Patients with non-resectable disease or with a PCI $>23$ underwent palliative surgery, if needed.

$F D G-P E T / C T$. All patients were imaged on a dedicated BGO PET system coupled to a helical CT (Discovery LS; GE Healthcare Technologies; Milwaukee, WI, USA). Whole-body imaging was performed according to routine standard practice guidelines (18). PET imaging was started between 60 and 75 minutes after intravenous administration of 250 to $350 \mathrm{MBq}{ }^{18} \mathrm{FDG}$, according to the body mass index of the patient. The CT scan was performed at 'low-dose' without oral or intravenous contrast administration. Before FDG injection, the patient's blood sugar level was routinely checked and registered. All images were reconstructed using a standard OSEM algorithm. All PET/CT images were visualized on a dedicated viewing station (Advantage Windows 4.3; General Electric, Milwaukee, WI, USA). Co-registered PET/CT scans were displayed using a standard grey scale for the CT images and a coloured amber scale for the PET data.

FDG-PET/CT review. A unique anonymous PET imaging database was created which randomly mixed up the patients from groups A and B using Excel software (Microsoft Corporation, Washington, USA). Nuclear medicine PET imaging specialists re-assessed all $\mathrm{PET}$ and fused PET/CT images in batch. Both were completely blinded to the results of the other observer, and to the medical records of the patients. Only images of the abdomen were assessed.
Analysis of sensitivity, specificity and predictive values. A preestablished patient-based scoring card for PET/CT image analysis was used, which listed the following categories: a) normal, no signs of peritoneal carcinomatosis; b) diffuse or moderate FDG uptake, probably benign (mural bowel uptake); c) abnormal FDG uptake, probably tumoural (focal uptake in the wall of the bowel, diffuse hypermetabolism in peritoneal areas without clear anatomical CT correlates); or d) definite recurrence or metastases (PET lesion correlating with distinct anatomical CT nodule).

The score cards of both independent observers were compared and a consensus was obtained. In cases of persistent inter-observer discrepancy, a third nuclear medicine specialist acted as a referee for the final decision and consensus. The PET/CT classifications were then regrouped with ' $a$ ' and ' $b$ ' being classed as negative, and ' $c$ ' and 'd' classed as positive for tumour recurrence. Only PET CT-positivity was assessed for correlation with surgical PCI classification.

Both histological and surgical findings served as the standard of reference for the diagnosis of PC. The sensitivity, specificity, and positive (PPV) and negative (NPV) predictive values and accuracy were determined for each patient.

Correlation between surgical and PET/CT PCI. The Sugarbaker PCI score was used as the frame of reference for the description of the extent of peritoneal dissemination at surgical exploration (surgical PCI score).

No standard scoring system exists for the evaluation of PC on $\mathrm{PET} / \mathrm{CT}$. In concordance with the scoring system used for surgical evaluation (PCI), we arbitrarily used a similar classification, with the only difference being that regions 9 to 12 were compressed. In practice, it was judged to be too difficult to differentiate a nodule located on the small intestine from one on the parietal peritoneum. Hence, the peritoneal cavity was divided into nine regions for the FDG-PET CT analysis (Figure 1).

Furthermore, we classified PC according to PET metabolic activity and topography. The intensity evaluation (metabolic activity) of foci was compared with the intensity in the liver. The following classification was used: A) lower intensity; B) equal intensity; or C) higher intensity. For the topographic description, we used the following classification: i) nodular (less than $1 \mathrm{~cm}$ in diameter, one or more); ii) mass (more than $1 \mathrm{~cm}$ in diameter, one or more); and iii) diffuse (no nodular lesion discerned).

Finally, the FDG-PET/CT score was compared with the surgical PCI score. To compare the two different scoring systems, we only considered the most metabolically active quadrant on the PET scoring card and the highest scored quadrant in the surgical PCI score.

Statistical analyses. The sensitivity, specificity, PPV, NPV and accuracy of FDG-PET/CT for PC detection were calculated using standard definitions (19).

\section{Results}

Fifty-two patients were included in this study. There were 23 males and 29 females. The mean age was 57.7 (range=2684) years. Patient demographic and clinical characteristics are reported in Table I.

The mean delay between FDG-PET/CT and surgery for patients who underwent surgical exploration (Group A) was 30 (range=6-40) days. The PC diagnosis was synchronous in 


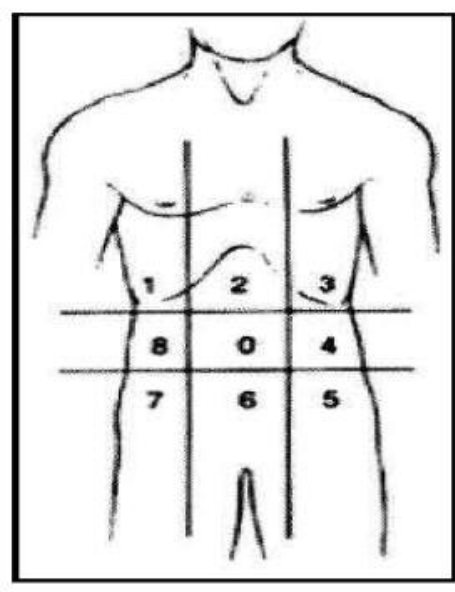

Figure 1. Quadrant distribution for scoring.

$65 \%(17 / 26)$ and metachronous in $35 \%(9 / 26)$ of cases. In the surgical group, 22 patients were treated with cytoreductive surgery associated with HIPEC, and four underwent exploratory surgery, but were excluded due to further cytoreductive surgery or underwent palliative surgery. Three out of 26 patients presented with associated extraperitoneal metastases (hepatic).

The sensitivity of FDG-PET/CT was $85 \%$ (22/26). There were four false-negative results. All four of these patients presented with mucinous adenocarcinoma, and three had a low PCI score with small lesions found during surgical exploration. The specificity was $88.5 \%$ (23/26). There were three false-positive results, all related to colonic physiological metabolic activity. The PPV was $88.5 \%$ $(23 / 26)$ and the NPV was $85 \%(22 / 26)$. The accuracy was $87 \%(45 / 52)$. The intensity and topographic evaluation of PC on FDG-PET/CT is reported in Table II.

The most involved and highest scored quadrant on FDGPET/CT corresponded to the most scored quadrant at surgery in $65.4 \%(17 / 26)$. Considering only those patients with a positive $\mathrm{PET} / \mathrm{CT}$ for $\mathrm{PC}$, the correspondence was $77.3 \%$ $(17 / 22)$. In five patients, the PCI score of the corresponding most metabolically active area detected on FDG-PET/CT was not maximal in comparison with other involved areas.

\section{Discussion}

Our study shows that FDG-PET/CT is a sensitive (85\%) and specific $(88 \%)$ tool in the detection of PC of colorectal origin. In addition, we observed a relatively good correlation (77\%) between the most metabolically active area on FDG$\mathrm{PET} / \mathrm{CT}$ and the most scored area on the surgical PCI score.

Locoregional treatment combining cytoreductive surgery with HIPEC has impressively improved the prognosis of
Table I. Demographic and clinical characteristics of patients included in this study.

\begin{tabular}{llc}
\hline Patients, n (\%) & Total & 52 \\
& CRC with PC & $26(50)$ \\
& CRC without PC & $26(50)$ \\
Gender, n & Male & 23 \\
& Female & 29 \\
Age (years) & Mean (range) & $57.7(26-84)$ \\
Tumour location, n (\%) & Colon & $31(60)$ \\
& Rectum & $16(31)$ \\
& Appendix & $5(9)$ \\
Delay FDG-PET/computed & Mean (range) & $30(6-40)$ \\
tomographic to surgery (days)* & & \\
PC, n (\%)* & Synchronous & $17(65)$ \\
& Metachronous & $9(35)$ \\
Treatment, n (\%)* & HIPEC & $22(85)$ \\
& Exploration & $4(15)$ \\
Extra-peritoneal metastases, n $(\%) *$ & Presence & $3(11.5)$ \\
& Absence & $23(88.5)$ \\
\hline
\end{tabular}

CRC, Colorectal cancer; PC, peritoneal carcinomatosis; FDG-PET, ${ }^{18} \mathrm{~F}$ fluorodeoxyglucose positron-emission tomography; CT, computed tomography; HIPEC, hyperthermic intra-peritoneal chemotherapy. *Concerns only patients with PC (surgical group).

Table II. Topographic and metabolic intensity classification of patients with peritoneal carcinomatosis.

\begin{tabular}{lccc}
\hline Intensity classification $^{\mathrm{a}}$ & Nodular & Mass & Diffuse \\
\hline Lower & 0 & 0 & 2 \\
Equivalent & 0 & 0 & 0 \\
Higher & $12^{*}$ & $9^{*}$ & $4^{\dagger}$ \\
\hline
\end{tabular}

aCompared to that of the liver. *Four patients had a combination of diffuse and nodular disease in different peritoneal cancer index (PCI) regions. ${ }^{\dagger}$ One patient had a combination of diffuse and nodular disease in different PCI regions.

patients with PC of colorectal origin. In a French multicentric study, 5-year survival was reported at $27 \%$ with a median survival of 30 months (20). Nevertheless, this therapeutic modality is associated with a risk of morbidity and mortality. In trained surgical teams, the 1-month grade 3-4 morbidity and mortality were reported as $31-43 \%$ and $3 \%$, respectively $(20,21)$. Therefore, a major concern in the management of patients with PC of colorectal origin is the selection of those who will benefit from cytoreductive surgery and HIPEC (2). In our experience, we note that about one-sixth of patients who seemed to be candidates for cytoreductive surgery and HIPEC were excluded during exploratory surgery, although preoperative imaging was unable to predict the extent of intraperitoneal disease. In fact, the complete resection of peritoneal nodules is one of the 
major prognostic factors for survival, and depends on both the extent and location of the PC. Various clinical predictive factors and CT scan criteria have been reported for better selection of patients $(22,23)$.

The evaluation of PC using abdominal CT scanning is difficult. Various authors have evaluated the diagnostic performance of abdominal CT with double contrast, and the greatest limitation is the spatial definition for small peritoneal lesions and the low performance in detecting nodules located on the mesentery, small bowel serosa, and pelvis $(22,24,25)$. De Bree et al. reported a sensitivity of $67 \%$ and $18 \%$ for nodules more than $5 \mathrm{~cm}$ and less than $1 \mathrm{~cm}$, respectively (25). Data concerning the role of magnetic resonance imaging in the detection of PC are poor. Kim et al. reported recently that the sensitivity of magnetic resonance imaging was $86 \%$ compared with $43 \%$ for FDG-PET/CT in the detection of PC in recurrent ovarian cancer (26).

Although the role of FDG-PET in the diagnosis of various tumours has been demonstrated, the role of FDG-PET in the diagnosis of PC remains unclear. Turklow et al. compared the performance of CT scan (simple contrast) with that of FDG-PET in a series of 24 patients with PC of various origins. The sensitivity was $57 \%$ for FDG-PET and $42 \%$ for CT (27). Dromain et al. evaluated and compared the performance of double contrast CT scans with that of FDGPET in the diagnostic work up of patients with PC of gastrointestinal origin. They found that the performance of FDG-PET was not superior to that of CT. In fact, the sensitivity of PET and CT in the detection of PC was $52 \%$ and $82 \%$, respectively. Furthermore, PET underestimated the extent of peritoneal involvement (14).

Data are limited regarding the performance of $\mathrm{CT}$ compared to that of the FDG-PET/CT in the detection of PC of colorectal origin $(15,28)$. Pfannenberg et al. recently reported the superiority of FDG-PET/CT to that of FDG-PET alone, with a sensitivity of $88 \%$ and $63 \%$, respectively, in patients with PC of ovarian origin (28). Pasquali et al. reported a sensitivity of $91 \%$ and $82 \%$ in PC detection with CT and FDG-PET/CT, respectively (15).

In our study, FDG-PET/CT had a sensitivity of $85 \%$, which is consistent with the results of previous studies. It is important to note that in our study, each imaging series was analysed by two experienced nuclear medicine physicians, with the intervention of a third in cases of discordant assessment.

The four false-negative FDG-PET/CT results occurred in patients with mucinous tumours. Poor results in patients with this type of histology have been described in the literature, and are probably based on the low cellularity of these tumours, which results in a level of accumulation of FDG that is too low to be detected by the PET system $(29,30)$. Furthermore, for three patients, the nodules described during surgery were small. A low spatial resolution combined with low metabolic activity can explain these findings. Only one patient had a high PCI score and a negative FDG-PET/CT exclusively related to low metabolic activity and low FDG avidity of a mucinous tumour.

The second motivation for performing this study was to evaluate the eventual role of FDG-PET/CT in the therapeutic management of patients with PC of colorectal cancer origin. In fact, there is no standardized algorithm for the management of PC of colorectal origin. Frontline surgery is often proposed because chemotherapy is ineffective in the treatment of PC of colorectal origin. Koppe et al. reported a median survival of 5 to 12 months with systemic 5fluorouracil-based chemotherapy (31). Recently, the therapeutic arsenal for treating patients with colorectal cancer has evolved with the advent of new chemotherapeutic agents such as oxaliplatin and irinotecan, and monoclonal antibodies such as bevacizumab. These new agents significantly improve the prognosis of patients (32). The use of neoadjuvant chemotherapy could be proposed as an option in the therapeutic management of PC patients. However, a well-known difficulty in PC is the evaluation of the response to chemotherapy in the absence of tumoural marker elevation. Hendlisz et al. have reported the role of PET/CT to assess early chemotherapeutic response in metastatic colorectal cancer showing that only $43 \%$ of metabolically responding patients had a response according to Response Evaluation Criteria in Solid Tumors (33). Furthermore, an ongoing study is evaluating early therapeutic response in the adjuvant setting of primary colorectal cancer (34). Therefore, FDG-PET/CT could help evaluate the metabolic response to chemotherapeutic treatment and allow for better selection of candidates for surgery as a function of their response. Nevertheless, there is no actual proof that the most metabolically active nodule in the peritoneal cavity corresponds to the most involved area identified during exploratory surgery. It would make no sense to treat patients with small hypermetabolic peritoneal nodules who respond to chemotherapy by FDG-PET/CT but who also present with major non-metabolic and non-assessable peritoneal nodules. Therefore, one of the major findings of our study was to confirm that there is a good correlation (77.3\%) between FDG-PET/CT findings and the corresponding PCI score. The five patients in whom the most metabolically active areas were not correlated with the most affected area on surgical PCI score are explained by the poor accuracy of PET/CT to estimate PC quantification (15). In any case, the results of this study justify further studies on the use of FDG-PET/CT in evaluating the response to neoadjuvant chemotherapy in patients with PC of colorectal origin, which could be essential for better preselection of patients for whom further cytoreductive surgery and HIPEC could result in a prognostic benefit.

In conclusion, FDG-PET CT might play a role in the selection of patients with PC of non-mucinous colorectal 
cancer origin who are candidates for cytoreductive surgery and HIPEC. The good correlation between the most metabolically active quadrant on FDG-PET/CT and the most affected area based on PCI score could position FDG$\mathrm{PET} / \mathrm{CT}$ as a promising tool in the evaluation of chemotherapeutic response in patients with PC treated by neoadjuvant chemotherapy and be useful for guiding therapeutic management of patients with $\mathrm{PC}$ of CRC origin.

\section{Acknowledgements}

The Authors acknowledge the contribution of a medical writer, Sandy Field, Ph.D., for English language editing and formatting of the article.

\section{References}

1 Jayne DG, Fook S, Loi C and Seow-Choen F: Peritoneal carcinomatosis from colorectal cancer. Br J Surg 89: 1545-1550, 2002.

2 Elias D and Ouellet JF: Intraperitoneal chemohyperthermia. Rationale, technique, indications and results. Surg Oncol Clin North Am 10: 915-933, 2001.

3 Minsky BD, Mies C, Rich TA, Recht A and Chaffey JT: Potentially curative surgery of colon cancer: Patterns of failure and survival. J Clin Oncol 6: 106-118, 1988.

4 Sugarbaker PH, Schellinx ME, Chang D, Koslowe P and von Meyerfeldt M: Peritoneal carcinomatosis from adenocarcinoma of the colon. World J Surg 20: 585-592, 1996.

5 Elias D, Blot F, El Otmany A, Antoun S, Lasser P, Boige V, Rougier $\mathrm{P}$ and Ducreux M: Curative treatment of peritoneal carcinomatosis arising from colorectal cancer by complete resection and intraperitoneal chemotherapy. Cancer 92: 71-76, 2001.

6 Liberale G, Pocard M, Manganas D, Ducreux M, Lasser P and Elias D: Hyperthermic intraperitoneal chemotherapy in the treatment of peritoneal carcinomatosis of digestive and peritoneal origin: Rationale. Acta Chir Belg 106: 291-296, 2006.

7 Elias D, Lefevre H, Chevalier J, Brouquet A, Marchal F, Classe JM, Ferron G, Guilloit JM, Meeus P, Goéré D and Bonastre J: Complete cytoreductive surgery plus intraperitoneal chemohyperthermia with oxaliplatin for peritoneal carcinomatosis of colorectal origin. J Clin Oncol 27: 681-685, 2009.

8 Denis B and Elias D: Prise en charge symptomatique de la carcinose péritonéale. Gastroenterol Clin Biol 28: 17-25, 2004.

9 Koh JL, Yan TD, Glenn D and Morris DL: Evaluation of preoperative computed tomography in estimating peritoneal cancer index in colorectal peritoneal carcinomatosis. Ann Surg Oncol 16: 327-333, 2009.

10 Suzuki A, Kawano T, Takahashi N, Lee J, Nakagami Y, Miyagi E, Hirahara F, Togo S, Shimada $\mathrm{H}$ and Inoue T: Value of ${ }^{18} \mathrm{~F}$ FDG PET in the detection of peritoneal carcinomatosis. Eur $\mathrm{J}$ Nucl Med Mol Imaging 31: 1413-1420, 2004.

11 Mangili G, Picchio M, Sironi S, Viganò R, Rabaiotti E, Bornaghi D, Bettinardi V, Crivellaro C, Messa C and Fazio F: Integrated $\mathrm{PET} / \mathrm{CT}$ as a first-line re-staging modality in patients with suspected recurrence of ovarian cancer. Eur J Nucl Med Mol Imaging 34: 658-666, 2007.
12 Papathanassiou D, Bruna-Muraille C: Positron emission tomography in oncology: Present and future of PET and PET/CT. Crit Rev Oncol Hematol 72: 239-254, 2009.

13 Aukema TS, Rutgers EJ, Vogel WV, Teertstra HJ, Oldenburg HS, Vrancken Peeters MT, Wesseling J, Russell NS and Valdés Olmos RA: The role of FDG PET/CT in patients with locoregional breast cancer recurrence: a comparison to conventional imaging techniques. Eur J Surg Oncol 36: 387-392, 2010.

14 Dromain C, Leboulleux S, Auperin A, Goere D, Malka D, Lumbroso J, Schumberger M, Sigal R and Elias D: Staging of peritoneal carcinomatosis: enhanced CT vs. PET/CT. Abdom Imaging 33: 87-93, 2008.

15 Pasqual EM, Bertozzi S, Bacchetti S, Londero AP, Basso SM, Santeufemia DA, Lo Re G and Lumachi F: Preoperative assessment of peritoneal carcinomatosis in patients undergoing hyperthermic intraperitoneal chemotherapy following cytoreductive surgery. Anticancer Res 34: 2363-2368, 2014.

16 Jacquet $\mathrm{P}$ and Sugarbaker PH: Clinical research methodologies in diagnosis and staging of patients with peritoneal carcinomatosis. Cancer Treat Res 82: 359-374, 1996.

17 Elias D, Liberale G, Manganas D, Lasser P and Pocard M: Surgical treatment of peritoneal carcinomatosis. Ann Chir 129: 530-533, 2004

18 Bombardieri E, Aktolun C, Baum RP, Bishof-Delaloye A, Buscombe J, Chatal JF, Maffioli L, Moncayo R, Mortelmans L and Reske SN: FDG-PET procedures guidelines for tumor imaging. Eur J Nucl Med Mol Imaging 30: 115-124, 2003.

19 Beck JR: Likelihood ratios. Another enhancement of sensitivity and specificity. Arch Pathol Lab Med 110: 685-686, 1986.

20 Elias D, Gilly F, Boutitie F, Quenet F, Bereder JM, Mansvelt B, Lorimier G, Dubè $\mathrm{P}$ and Glehen $\mathrm{O}$ : Peritoneal colorectal carcinomatosis treated with surgery and perioperative intraperitoneal chemotherapy: retrospective analysis of 523 patients from a multicentric French study. J Clin Oncol 28: 6368, 2010.

21 Chua TC, Saxena A, Schellekens JF, Liauw W, Yan TD, Fransi S, Zhao J and Morris DL: Morbidity and mortality outcomes of cytoreductive surgery and perioperative intraperitoneal chemotherapy at a single tertiary institution: towards a new perspective of this treatment. Ann Surg 251: 101-106, 2010.

22 Jacquet P, Jelinek JS, Steves MA and Sugarbaker PH: Evaluation of computed tomography in patients with peritoneal carcinomatosis. Cancer 72: 1631-1636, 1993.

23 Elias D, Benizri E, Vernerey D, Eldweny H, Dipietrantonio D and Pocard M: Preoperative criteria of incomplete resectability of peritoneal carcinomatosis from non-appendiceal colorectal carcinoma. Gastroenterol Clin Biol 29: 1010-1013, 2005.

24 Coakley FV, Choi PH, Gougoutas CA, Pothuri B, Venkatraman E, Chi D, Bergman A and Hricak H: Peritoneal metastases: detection with spiral CT in patients with ovarian cancer. Radiology 223: 495-499, 2002.

25 De Bree E, Koops W, Kröger R, van Ruth S, Witkamp AJ and Zoetmulder FA: Peritoneal carcinomatosis from colorectal or appendiceal origin: correlation of preoperative CT with intraoperative findings and evaluation of interobserver agreement. J Surg Oncol 86: 64-73, 2004.

26 Kim CK, Park BK, Choi JY, Kim BG and Han H: Detection of recurrent ovarian cancer at MRI: comparison with integrated PET/CT. J Comput Assist Tomogr 31: 868-875, 2007. 
27 Turlakow A, Yeung HW, Salmon AS, Macapinlac HA and Larson SM: Peritoneal Carcinomatosis: Role of ${ }^{18} \mathrm{~F}-\mathrm{FDG}$ PET. J Nucl Med 44: 1407-1412, 2003.

28 Pfannenberg C, Königsrainer I, Aschoff P, Oksüz MO, Zieker D, Beckert S, Symons S, Nieselt K, Glatzle J, Weyhern CV, Brücher BL, Claussen CD and Königsrainer A: ${ }^{18}$ F-FDG-PET/CT to select patients with peritoneal carcinomatosis for cytoreductive surgery and hyperthermic intraperitoneal chemotherapy. Ann Surg Oncol 16: 1295-1303, 2009.

29 Stahl A, Ott K, Weber WA, Becker K, Link T, Siewert JR, Schwaiger $M$ and Fink U: FDG PET imaging of locally advanced gastric carcinomas: correlation with endoscopic and histopathological findings. Eur J Nucl Med 30: 288-295, 2003.

30 Nishiyama Y, Yamamoto Y, Monden T, Sasakawa Y, Tsutsui K, Wakabayashi $\mathrm{H}$ and Ohkawa $\mathrm{M}$ : Evaluation of delayed additional FDG PET imaging in patients with pancreatic tumour. Nucl Med Commun 26: 895-901, 2005.

31 Koppe MJ, Boerman C, Oyen WJ and Bleichrodt RP: Peritoneal carcinomatosis of colorectal origin incidence and current treatment strategies. Ann Surg 243: 212-222, 2006.

32 Folprecht G, Lutz M, Rougier P: Systemic chemotherapy in patients with known peritoneal carcinomatosis from colo-rectal cancer. Proc Gastrointestinal Canc Symp 134: 425-440, 2007.
33 Hendlisz A, Golfinopoulos V, Garcia C, Covas A, Emonts P, Ameye L, Paesmans M, Deleporte A, Machiels G, Toussaint E, Vanderlinden B, Awada A, Piccart M and Flamen P: Serial FDG-PET/CT for early outcome prediction in patients with metastatic colorectal cancer undergoing chemotherapy. Ann Oncol 23: 1687-1693, 2012.

34 Hendlisz A, Golfinopoulos V, Deleporte A, Paesmans M, Mansy HE, Garcia C, Peeters M, Annemans L, Vandeputte C, Maetens M, Borbath I, Dresse D, Houbiers G, Fried M, Awada A, Piccart M, Laethem JL and Flamen P: Preoperative chemosensitivity testing as predictor of treatment benefit in adjuvant stage III colon cancer (PePiTA): Protocol of a prospective BGDO (Belgian Group for Digestive Oncology) multicentric study. BMC Cancer 13: 190, 2013. 